$\Rightarrow$ HAEMATOLOGICAL CANCER

\section{Acalabrutinib - a new option in CLL}

Patients with chronic lymphocytic leukaemia (CLL) can derive benefit from the BTK inhibitor ibrutinib, alone or in combination with anti-CD20 antibodies (such as rituximab and obinutuzumab). Now, results from the phase III ELEVATE-TN trial indicate that acalabrutinib, another BTK inhibitor, is a new treatment option for these patients.

In ELEVATE-TN, patients with untreated CLL, who were aged $\geq 65$ years, or $18-65$ years and with comorbidities, were randomly allocated to receive acalabrutinib, either alone $(n=179)$ or in combination with obinutuzumab $(n=179)$, or obinutuzumab plus the cytotoxic agent chlorambucil $(n=177)$. After a median of 28.3 months, median progression-free survival (PFS) durations were not reached (NR) with acalabrutinib combination and monotherapy, and 22.6 months with chlorambucil combination (HR 0.1, 95\% CI 0.06-0.17; $P<0.0001$ and HR 0.2, 95\% CI 0.13-0.30; $P<0.0001$ for acalabrutinib combination and monotherapy, respectively, versus chlorambucil combination). At 24 months, PFS estimates were $93 \%, 87 \%$ and $43 \%$, respectively. This PFS benefit was consistent across patient subgroups.

Overall response rates were $94 \%$, $86 \%$ and $79 \%$ with acalabrutinib combination, monotherapy and chlorambucil combination, respectively $(P<0.0001$ and $P<0.08$ for acalabrutinib combination and monotherapy, respectively, versus chlorambucil combination). Of these responses, $13 \%, 1 \%$ and $5 \%$, respectively, were complete responses.

The incidence of grade $\geq 3$ adverse events (AEs) was $70.2 \%, 49.7 \%$ and $69.8 \%$ in patients receiving acalabrutinib combination, monotherapy and chlorambucil combination, respectively, most commonly neutropenia $(30 \%, 9 \%$ and $41 \%$, respectively). AE-related treatment discontinuation occurred in $11 \%$, $9 \%$ and $14 \%$ of patients, respectively.

In November 2019, the FDA approved acalabrutinib for adults with CLL or small lymphocytic lymphoma (SLL), regardless of age and comorbidities, in a decision partly based on the results from ELEVATE-TN. Several agents are currently approved for patients with CLL, and over the past few years researchers have aimed to determine the best regimen for different subgroups. In the phase III iLLUMINATE trial, involving a patient population similar to that in ELEVATE-TN, median PFS durations were NR and 19.0 months with obinutuzumab plus either ibrutinib or chlorambucil (HR 0.23; $P<0.0001$ ); 30 -month PFS was $79 \%$ versus $31 \%$. Results from trials comparing acalabrutinib versus ibrutinib in patients with CLL are eagerly awaited.

Diana Romero

ORIGINAL ARTICLE Sharman, J. P. et al. Lancet

395, 1278-1291 (2020)

RELATED ARTICLE Moreno, C. et al. Lancet Oncol. 20, 43-56 (2019)

\section{In the news}

\section{FROM AACR 2020 (PART 1)}

Like many events, this year's annual meeting of the American Association for Cancer Research (AACR), which was originally intended to take place on April 24-29, was cancelled owing to the coronavirus disease 19 (COVID-19) pandemic. Nonetheless, noting the continued importance of cancer research and treatment, even during a pandemic, the organizing committee took the decision to hold an abbreviated and entirely

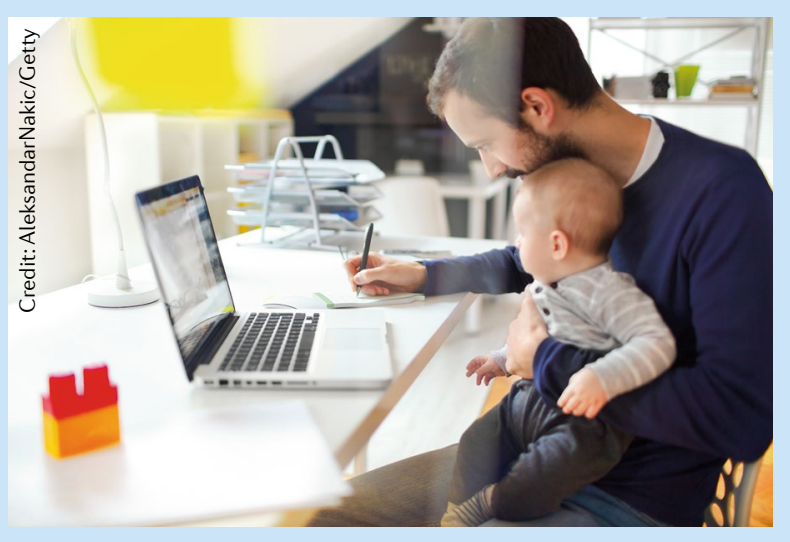

virtual meeting on April 28-29, focussing on clinical data. Owing to the lack of a registration fee, accommodation or travel costs, $>60,000$ people were able to register and 'attend' the meeting.

The most memorable session from this year's meeting will likely be the clinical plenary on COVID-19 and cancer. This session featured new data on the clinical experiences of treating patients with cancer and COVID-19 from hospitals in several parts of China including Wuhan, 12 de Octubre Hospital, Madrid, Gustave Roussy Cancer Centre, Paris, and data from TERAVOLT, an international lung cancer registry set up specifically for patients with COVID-19. Most, but not all, experiences confirmed what previous reports have suggested: patients with cancer seem to have worse COVID-19 outcomes than those without cancer. Other key conclusions are that patients with lung cancer and those with haematological malignancies and/or stage IV disease are most vulnerable. Among treatment-specific effects, associations between chemotherapy or surgery and worse COVID-19 outcomes were suggested by some but not all data; radiotherapy was not associated with worse COVID-19 outcomes.
Elsewhere, early data from KEYNOTE-555 indicated that patients with advanced-stage melanoma can benefit from 6-weekly, $400 \mathrm{mg}$ doses of pembrolizumab (objective response rate $39 \%$ among the first 44 patients). This announcement was closely followed by FDA accelerated approval of this dosing schedule.

Other notable clinical findings included data from the I-SPY 2 trial, indicating improved pathological response rates in the neoadjuvant setting for women with triple-negative or HER2-positive breast cancer. Significant improvements in progression-free survival were reported in patients with advanced-stage melanoma in the IMspire 150 and SWOG S1320 trials. Finally, data from IMbassador250 revealed no significant improvement in overall survival with the addition of atezolizumab to enzalutamide in men with metastatic castration-resistant prostate cancer.

A second virtual annual meeting, featuring the full range of topics typically covered at AACR, will take place from June 22-24.

Peter Sidaway 